\title{
2016 Consensus statement on prevention of atherosclerotic cardiovascular disease in the Hong Kong population
}

\author{
Bernard MY Cheung *, CH Cheng, CP Lau, Chris KY Wong, Ronald CW Ma, Daniel WS Chu, \\ Duncan HK Ho, Kathy LF Lee, HF Tse, Alexander SP Wong, Bryan PY Yan, Victor WT Yan
}

\section{A B S T R A C T}

Introduction: In Hong Kong, the prevalence of atherosclerotic cardiovascular disease has increased markedly over the past few decades, and further increases are expected. In 2008, the Hong Kong Cardiovascular Task Force released a consensus statement on preventing cardiovascular disease in the Hong Kong population. The present article provides an update on these recommendations.

Participants: A multidisciplinary group of clinicians comprising the Hong Kong Cardiovascular Task Force-10 cardiologists, an endocrinologist, and a family physician-met in September 2014 and June 2015 in Hong Kong.

Evidence: Guidelines from the American College of Cardiology/American Heart Association, the European Society of Hypertension/European Society of Cardiology, and the Eighth Joint National Committee for the Management of High Blood Pressure were reviewed.

Consensus Process: Group members reviewed the 2008 Consensus Statement and relevant international guidelines. At the meetings, each topical recommendation of the 2008 Statement was assessed against the pooled recommendations on that topic from the international guidelines. A final recommendation on each topic was generated by consensus after discussion.

Conclusions: It is recommended that a formal risk scoring system should be used for risk assessment of all adults aged 40 years or older who have at least one cardiovascular risk factor. Individuals can be classified as having a low, moderate, or high risk of developing atherosclerotic cardiovascular disease, and appropriate interventions selected accordingly. Recommended lifestyle modifications include adopting a healthy eating pattern; maintaining a low body mass index; quitting smoking; and undertaking regular, moderate-intensity physical activity. Pharmacological interventions should be selected as appropriate after lifestyle modification.

\section{Hong Kong Med J 2017;23:191-201}

DOI: 10.12809/hkmj165045

BMY Cheung *, MB BChir (Cantab), PhD (Cantab)

${ }^{2} \mathrm{CH}$ Cheng, MB, BS

${ }^{3}$ CP Lau, MB, BS, MD

${ }^{4}$ CKY Wong, MB, ChB (Glasg)

${ }^{5}$ RCW Ma, MB BChir (Cantab)

2 DWS Chu, MB, BS (NSW)

${ }^{4}$ DHK Ho, MB, BS

${ }^{2}$ KLF Lee, MB, BS

${ }^{1}$ HF Tse, MD, PhD

${ }^{2}$ ASP Wong, MB, BS

${ }^{5}$ BPY Yan, MB, BS

${ }^{2}$ VWT Yan, MB, BS

Department of Medicine, The University of Hong Kong, Queen Mary Hospital, Pokfulam, Hong Kong

Private practice, Hong Kong

Institute of Cardiovascular Science and Medicine, The University of Hong Kong, Pokfulam, Hong Kong

${ }^{4}$ Hong Kong Sanatorium \& Hospital, Happy Valley, Hong Kong

${ }^{5}$ Department of Medicine and Therapeutics, The Chinese University of Hong Kong, Prince of Wales Hospital, Shatin, Hong Kong

* Corresponding author: mycheung@hku.hk

\section{Introduction}

Atherosclerotic cardiovascular disease (ASCVD), which includes coronary heart disease (CHD), peripheral vascular disease and stroke, is currently one of the most common causes of morbidity and mortality worldwide. ${ }^{1}$ Unfortunately the prevalence of ASCVD is expected to increase further over the next few decades due to a number of factors including an ageing population and increasing industrialisation. The latter is associated with increased exposure to known ASCVD risk factors such as smoking, low levels of physical activity, and poor dietary habits such as reduced consumption of fruit and vegetables and increased fat and salt intake. $^{2}$

In Hong Kong, the prevalence of ASCVD risk factors has increased markedly over the past few decades. For example, the 2005-2008 Hong Kong Cardiovascular Risk Factor Prevalence Study-3 (CRISPS-3) reported an $8.6 \%$ increase in the prevalence of abdominal obesity (waist circumference $\geq 90 \mathrm{~cm}$ in men and $\geq 80 \mathrm{~cm}$ in women) and a $21.5 \%$ 


\section{6年香港預防動脈䉼樣硬化性心血管疾病的 共識聲明}

張文勇、鄭俊豪、劉柱柏、王國耀、馬青雲、朱偉星、

何鴻光、李麗芬、謝鴻發、王壽鵬、甄秉言、甄榮達

引言：香港動脈粥樣硬化性心血管疾病的患病率在過去數十年顯著 增加, 預期患者人數還會進一步上升。香港心血管工作小組（Hong Kong Cardiovascular Task Force；HKCTF）於2008年發表了一份關 於香港預防動脈粥樣硬化性心血管疾病的共識聲明。本文提供了有關 建議的更新。

參與者：HKCTF是由 10 名心藏病學、1名內分泌學和 1 名家庭醫學專 科醫生組成的多學科小組。他們於 2014 年9月和 2015 年6月在香港舉 行會議。

證據：建議主要基於以下學會制定的指引：美國心臟病學會／美國心 藏協會、歐洲高血壓學會 / 歐洲心藏病學會和第八屆聯合全國高血壓 管理委員會。

共識過程：小組成員基於 2008 年的共識聲明以及相關國際指引進行審 查。會議上根據國際指引中的匯總建議來評估2008年發表的每一項共 識聲明。小組成員參與共識的討論制定, 以協商方式達成每一項推薦 建議。

結論：建議對所有 40 歲或以上至少有一項心血管危險因素的成年人， 利用正式的動脈粥樣硬化性心血管疾病的風險評估系統進行評估, 把 病人分為低度、中度或高度風險, 並選擇相應適當干預。所推薦的生 活方式改變包括培養健康飲食習慣、保持體重指數於低水平、戒菸、 並定期進行中等強度的體力運動。改變生活方式後才進行藥物治療。

increase in the prevalence of hypertension among a cohort of 1803 subjects recruited from CRISPS1 , the first such survey conducted between 1995 and $1996 .{ }^{3}$ Of the 551 participants of the Hong Kong Cardiovascular Task Force Risk Management Programme, $65.4 \%$ had hypertension, $63.7 \%$ dyslipidaemia, and $33.3 \%$ diabetes at baseline (BMY Cheung, unpublished data).

Global efforts are underway to promote ASCVD prevention and reduce the risk of major ASCVD events. These efforts have yielded benefitsbetween 1990 and 2013, a substantial reduction in cardiovascular mortality was seen in central Europe (5.2\%) and western Europe (12.8\%), attributed primarily to birth cohorts' decreased exposure to tobacco smoking, improvements in diet, improved treatment of cardiometabolic risk factors, and improved treatment of CVD. ${ }^{4}$

\section{Methods}

A multidisciplinary group of clinicians comprising the Hong Kong Cardiovascular Task Force-10 cardiologists, an endocrinologist, and a family physician-met in September 2014 and June 2015 in Hong Kong with the aim of updating the first
Consensus Statement on Preventing Cardiovascular Disease in the Hong Kong Population published in 2008. ${ }^{5}$ Prior to the consensus meetings, group members reviewed the 2008 Consensus Statement and relevant guidelines from the American College of Cardiology/American Heart Association, the European Society of Hypertension/European Society of Cardiology, and the Eighth Joint National Committee for the Management of High Blood Pressure, among others. ${ }^{5-9}$ At the meetings, each topical recommendation of the 2008 Statement was assessed against the pooled recommendations on that topic from the international guidelines reviewed. A final recommendation on each topic was generated by consensus after discussion.

The recommendations included in this consensus statement constitute the consensus opinion of the members of the Hong Kong Cardiovascular Task Force regarding the most appropriate interventions for the Hong Kong population.

\section{Recommendations}

\section{Risk assessment}

\section{Total cardiovascular risk}

Total ASCVD risk is based on the complex interactions of a number of different risk factors that together have a multiplicative effect. That is, the risk of ASCVD is amplified to a greater extent by the interaction of multiple risk factors than would be expected due to the cumulative effect of each risk factor alone. ${ }^{7,9}$ The present standard of practice for the primary prevention of ASCVD is to determine a patient's total ASCVD risk using a formal risk scoring algorithm. ${ }^{1,7,9}$

\section{Who to assess?}

In Hong Kong, it is recommended that ASCVD prevention efforts should be focused on adults aged 40 years or older who have at least one ASCVD risk factor. ${ }^{1,9}$ The total ASCVD risk should be formally calculated for such individuals, and they should receive ASCVD prevention advice and/or treatment according to their determined level of risk (high, moderate, or low). ${ }^{1,9}$ High-risk patients will benefit most from treatment and include:

- patients with overt ASCVD (CHD, previous myocardial infarction, previous stroke, or peripheral vascular disease) or those who are symptomatic (eg have experience with angina)

- patients with diabetes mellitus

- patients with one major ASCVD risk factor (eg moderate-to-severe hypertension, severely elevated lipid levels $)^{9}$

These patients automatically meet the threshold for intensive risk factor treatment and need not undergo formal risk scoring., 


\section{How to assess?}

It is important to note that the current recommendations do not espouse a preference for any particular method of risk projection, but recommend that formal ASCVD risk scoring should be performed for all potentially at-risk patients.

A patient's 10-year (total) risk for ASCVD may be calculated using a variety of methods. The most recently published algorithm uses the American Pooled Cohort Risk Assessment Equations that are sex-and race-specific estimates for African-American and White men and women aged 40 to 79 years. They utilise age, total and high-density lipoprotein (HDL) cholesterol, systolic blood pressure (BP), diabetes, and current smoking status to calculate the total ASCVD risk. ${ }^{1}$ The European Systematic Coronary Risk Evaluation (SCORE) system is another wellvalidated system that uses sex, age, systolic BP, total cholesterol, and current smoking status to predict the risk of fatal cardiovascular events. ${ }^{7}$ Another risk assessment system, QRISK2, includes the risk factor of 'self-assigned ethnicity' (including Chinese) in the computation $^{10,11}$; however, the model was validated for Chinese immigrants to the United Kingdom and, thus, its applicability to the local Chinese population is unknown.

The calculated risk score is used to stratify patients into low-, moderate-, and high-risk categories (Fig). When interpreting these scores, the clinician should bear in mind that they were developed and validated for western populations. In addition, it must be remembered that any calculated ASCVD score is simply an indicator of total cardiovascular risk. ${ }^{9}$ Although it can guide patient treatment, it cannot be a substitute for individualised patient evaluation and management. The clinician is advised to take all factors into account and to treat the patient individually rather than treat the risk score.

\section{Risk interventions}

There is robust scientific evidence that the development of ASCVD in at-risk patients may be slowed and/or prevented by lifestyle modification, reduction of metabolic risk factors, and pharmacological treatment. ${ }^{7,9-19}$ Listed below are

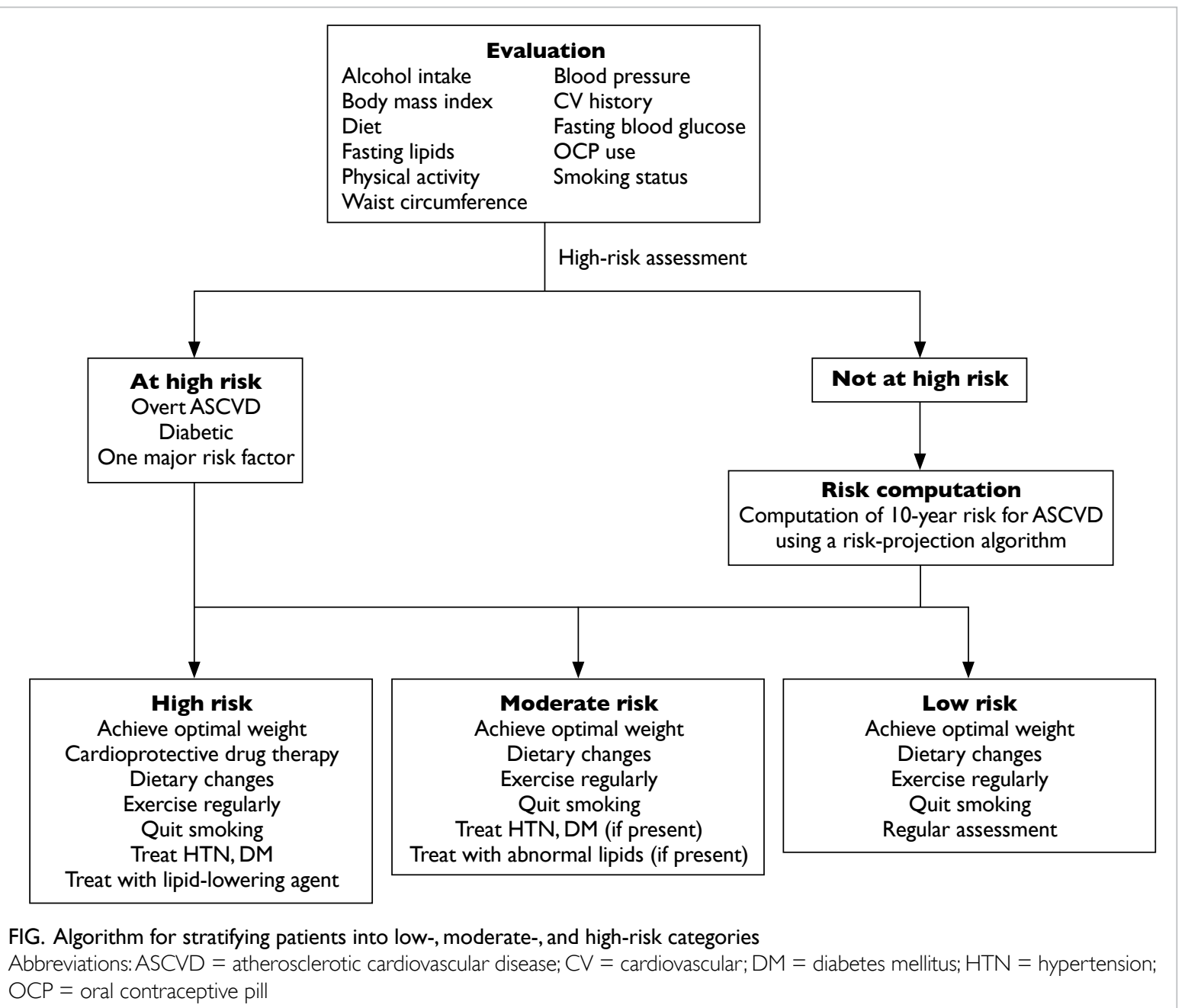


the major modifiable risk factors for ASCVD. The treatment goal is stated for each risk factor, along with general recommendations on how this goal may be achieved. Existing hypertension, ${ }^{7,8}$ dyslipidaemia, ${ }^{1,6}$ and diabetes ${ }^{20-22}$ treatment guidelines incorporate the latest evidence on how to treat these conditions and to what appropriate targets. The clinician is referred to these guidelines for further guidance.

\section{Diet}

Treatment goal: an overall healthy eating pattern

All patients at increased risk of ASCVD should be given advice and specific recommendations for eating a healthy diet. Advice should include?:

- matching energy intake with energy needs;

- eating a variety of fruits, vegetables, grains, lowor non-fat dairy products, legumes, fish, poultry, and lean meats;

- reducing saturated and trans fats to $<10 \%$ of total daily caloric intake, through replacement with polyunsaturated fats (vegetables, nuts, seeds, and seafood);

- reducing cholesterol intake;

- reducing salt intake; and

- limiting alcohol intake to no more than two drinks per day for men and one drink per day for women.

\section{Physical activity}

Treatment goal: a minimum of 30 minutes of moderate-intensity physical activity at least 5 times a week, or a minimum of 15 minutes of vigorousintensity physical activity at least 5 times a week ${ }^{9}$

- 'Moderate intensity' is defined as exercising at $64 \%$ to $76 \%$ of maximum heart rate (ie 220 minus age); activities include brisk walking, slow cycling, vacuuming, gardening, golf, tennis (doubles), ballroom dancing, and water aerobics. ' 'Vigorous intensity' is defined as exercising at $77 \%$ to $93 \%$ of maximum heart rate; activities include race walking, jogging or running, bicycling, heavy gardening, swimming laps, and tennis (singles). ${ }^{9}$

- The practice of the popular Chinese soft martial art tai chi may also be beneficial for individuals at risk of ASCVD. A systematic review has shown that tai chi has physiological and psychosocial benefits, and it also appears to be safe and effective in promoting flexibility, balance control, and cardiovascular fitness in older patients with chronic conditions. ${ }^{23}$

- All patients should consult their doctor prior to initiating graded exercise programmes.

\section{Overweight/obesity}

Treatment goal: maintenance of normal body mass index and waist circumference

- Normal body mass index is $18.5-22.9 \mathrm{~kg} / \mathrm{m}^{2}$ for
Asians, ${ }^{24}$ and normal waist circumference is $<90 \mathrm{~cm}$ (35.4 inches) for men and $<80 \mathrm{~cm}(31.5$ inches) for women. ${ }^{25}$

- Patients who are overweight or obese should strive to achieve normal body weight by restricting caloric intake and increasing physical activity.

- Drug therapy or surgical interventions may be a helpful adjunct for the treatment of severe obesity in some patients.

\section{Smoking}

\section{Treatment goal: complete smoking cessation}

- Assess the patient's tobacco use and strongly urge the patient to stop smoking.

- Determine the patient's degree of nicotine addiction and his/her readiness to quit smoking. For patients identified as willing to quit, a plan should be developed that may involve pharmacotherapy, counselling, cessation support mechanisms (eg follow-up calls and visits), and referral to specialised programmes, if available..$^{20,26}$

\section{Hypertension}

Risk factor reduction goal: blood pressure of $<140 / 90$ $\mathrm{mm} \mathrm{Hg}$ for the general population aged $<60$ years, including patients with previous stroke or transient ischaemic attack; patients with coronary heart disease; and patients with chronic kidney disease. For patients with diabetes, a target blood pressure of $<140 / 85 \mathrm{~mm} \mathrm{Hg}$ is recommended. For the general population aged $\geq 60$ years, a target blood pressure of $<150 / 90 \mathrm{~mm} \mathrm{Hg}$ is recommended $^{7-9}$

- Patients with a systolic BP of $\geq 130 \mathrm{~mm} \mathrm{Hg}$ or diastolic $\mathrm{BP}$ of $\geq 80 \mathrm{~mm} \mathrm{Hg}$ should be given advice and specific recommendations on reducing lifestyle risk factors.

- Patients who do not meet their primary goals as defined above should be given drug therapy tailored to their circumstances.

- The choice of first-line therapy is the prerogative of the attending physician. Suitable antihypertensive drugs include calcium channel blockers, angiotensin-converting enzyme (ACE) inhibitors, and angiotensin receptor blockers (ARBs), either alone or in combination (Tables 1 and 2). ${ }^{7,9}$ Diuretics (chlorthalidone and indapamide) and $\beta$-blockers may also be used, but their long-term use is associated with increased risk of new-onset diabetes ${ }^{30,31}$; of note, there is no evidence that hydrochlorothiazide-one of the most commonly prescribed antihypertensivesin its usual dose of 12.5 to $25 \mathrm{mg}$ daily reduces myocardial infarction, stroke, or death. ${ }^{32}$

- When target BP cannot be achieved with monotherapy or with a two-drug combination, 
TABLE I. Summary of blood pressure management guidelines $7,8,27-29$

\begin{tabular}{|c|c|c|c|c|}
\hline \multirow[t]{2}{*}{ Study group } & \multicolumn{4}{|c|}{ Recommended target BP (mm Hg) } \\
\hline & $\begin{array}{l}2011 \text { NICE Clinical Guideline } \\
\text { for the Management of } \\
\text { Hypertension, } 2009 \text { Clinical } \\
\text { Guideline for the Management } \\
\text { of Type } 2 \text { Diabetes, and } 2014 \\
\text { Clinical Guideline for the } \\
\text { Management of Chronic Kidney } \\
\text { Disease }\end{array}$ & $\begin{array}{l}2013 \text { ESH-ESC Task Force } \\
\text { Guideline for the Management } \\
\text { of Arterial Hypertension }\end{array}$ & $\begin{array}{l}2014 \text { JNC } 8 \\
\text { Guideline for the } \\
\text { Management of } \\
\text { High Blood Pressure } \\
\text { in Adults }\end{array}$ & $\begin{array}{l}2016 \text { Hong Kong } \\
\text { Cardiovascular } \\
\text { Task Force } \\
\text { Recommendations }\end{array}$ \\
\hline $\begin{array}{l}\text { General population (age } \\
\text { in years) }\end{array}$ & $\begin{array}{l}\text { Age }<80:<140 / 90 \\
\text { Age } \geq 80:<150 / 90\end{array}$ & $\begin{array}{l}\text { Age }<80:<140 / 90 \\
\text { Age } \geq 80:<140-150 / 90\end{array}$ & $\begin{array}{l}\text { Age }<60:<140 / 90 \\
\text { Age } \geq 60:<150 / 90\end{array}$ & $\begin{array}{l}\text { Age }<60:<140 / 90 \\
\text { Age } \geq 60:<150 / 90\end{array}$ \\
\hline Patients with diabetes & $\begin{array}{l}<140 / 80 \text { (<130/80 For people with } \\
\text { kidney, eye, or cardiovascular } \\
\text { damage) }\end{array}$ & $<140 / 85$ & $<140 / 90$ & $<140 / 85$ \\
\hline $\begin{array}{l}\text { Patients with chronic } \\
\text { kidney disease }\end{array}$ & $\begin{array}{l}<140 / 90 \text { (<130/80 In patients with } \\
\text { CKD and diabetes) }\end{array}$ & $\begin{array}{l}<140 / 90 \text { (systolic BP reading } \\
<140 \text { should be considered. } \\
\text { When overt proteinuria is present, } \\
\text { values }<130 \text { may be considered, } \\
\text { provided that changes in eGFR } \\
\text { are monitored) }\end{array}$ & $<140 / 90$ & $<140 / 90$ \\
\hline $\begin{array}{l}\text { Patients with CHD, ACS, } \\
\text { HF }\end{array}$ & Patients with CHD: $<140 / 90$ & $<140 / 90$ & Not specified & $<140 / 90$ \\
\hline $\begin{array}{l}\text { Other special populations } \\
\text { (age in years) }\end{array}$ & $\begin{array}{l}\text { Patients identified as having } \\
\text { a 'white coat' effect: consider } \\
\text { ambulatory or home BP } \\
\text { monitoring. Aim for usual target } \\
\text { BP during waking hours of: } \\
\text { Age }<80:<135 / 85 \\
\text { Age } \geq 80:<145 / 85\end{array}$ & $\begin{array}{l}\text { Patients with organ damage: } \\
<140 / 90\end{array}$ & Not specified & Not specified \\
\hline
\end{tabular}

Abbreviations: $\mathrm{ACS}=$ acute coronary syndrome; $\mathrm{BP}=$ blood pressure; $\mathrm{CHD}=$ coronary heart disease; $\mathrm{CKD}=$ chronic kidney disease; $\mathrm{eGFR}=$ estimated glomerular filtration rate; ESH-ESC = European Society of Hypertension/European Society of Cardiology; HF = heart failure; JNC 8 = Eighth Joint National Committee; NICE = National Institute for Health and Care Excellence

doses can be increased; if target BP cannot be achieved by a two-drug combination at full doses, switching to another two-drug combination, or adding a third drug, may be considered..$^{7-9}$ In patients with uncontrolled BP despite treatment with maximally tolerated doses of three antihypertensive medications, addition of the aldosterone antagonist spironolactone has achieved larger reductions in systolic BP than addition of the $\beta$-blocker bisoprolol, the alphaadrenergic blocker doxazosin, or placebo. ${ }^{33}$

\section{Dyslipidaemia}

Risk factor reduction goal: low-density lipoprotein cholesterol level of $<3 \mathrm{mmol} / \mathrm{L}$. For patients with overt atherosclerotic cardiovascular disease, the target level should be $<1.8 \mathrm{mmol} / \mathrm{L}^{9}$

- Low-density lipoprotein cholesterol (LDL-C) reduction decreases cardiovascular events. ${ }^{9}$

- Recommended target LDL-C level for patients stratified by ASCVD risk is as follows:

o Very high ASCVD risk: LDL-C $<1.8 \mathrm{mmol} / \mathrm{L}$, or $\mathrm{a} \geq 50 \%$ reduction if the baseline is between 1.8 and $3.5 \mathrm{mmol} / \mathrm{L}$ (Table 3 )

o High ASCVD risk: LDL-C $<2.6 \mathrm{mmol} / \mathrm{L}$, or a $\geq 50 \%$ reduction if the baseline is between 2.6 and $5.1 \mathrm{mmol} / \mathrm{L}$

o Low-to-moderate ASCVD risk: LDL-C <3.0 $\mathrm{mmol} / \mathrm{L}^{9}$

- Patients at low and moderate risk should be given advice and specific recommendations on lowering LDL-C through dietary adjustments, increased physical activity, and weight reduction. If the target is not met after 6 months, they should be given a lipid-lowering agent (Tables 4 and 5$).{ }^{35}$

- Patients at high risk should immediately be started on lipid-lowering therapy with a high-intensity statin (Tables 4 and 5). ${ }^{35}$ Importantly, however, pharmacokinetic studies have shown that Chinese patients achieve a higher plasma concentration of statin compared with Caucasians, and this may be associated with an increased risk of adverse effects. ${ }^{36}$ Consequently, the maximum approved doses of the statins available in Asia are around half the maximum approved doses in the United States. ${ }^{37}$ The clinician should, therefore, exercise caution when prescribing high-intensity statin therapy.

- Inhibitors of proprotein convertase subtilisin/ kexin type 9 have recently been approved for use 
TABLE 2. Classes of antihypertensive drugs

\begin{tabular}{|c|c|c|c|c|c|}
\hline Drug class & $\begin{array}{l}\text { Agents available in } \\
\text { Hong Kong }\end{array}$ & Mechanism of action & $\begin{array}{l}\text { Conditions favouring } \\
\text { use of drug class }\end{array}$ & $\begin{array}{l}\text { Compelling } \\
\text { contra-indications }\end{array}$ & $\begin{array}{l}\text { Possible contra- } \\
\text { indications }\end{array}$ \\
\hline ACE inhibitors & $\begin{array}{l}\text { Captopril } \\
\text { Enalapril } \\
\text { Imidapril } \\
\text { Lisinopril } \\
\text { Perindopril } \\
\text { Quinapril } \\
\text { Ramipril }\end{array}$ & $\begin{array}{l}\text { Blocks conversion } \\
\text { of angiotensin I to } \\
\text { angiotensin II by } \\
\text { inhibiting ACE }\end{array}$ & $\begin{array}{l}\text { Congestive heart } \\
\text { failure, post-MI } \\
\text { patients with reduced } \\
\text { LV ejection fraction, } \\
\text { diabetes mellitus, renal } \\
\text { dysfunction }\end{array}$ & $\begin{array}{l}\text { Pregnancy, } \\
\text { hyperkalaemia, } \\
\text { bilateral renal artery } \\
\text { stenosis }\end{array}$ & - \\
\hline Alpha-blockers & $\begin{array}{l}\text { Doxazosin } \\
\text { Prazosin }\end{array}$ & $\begin{array}{l}\text { Blocks } \alpha 1 \text {-adrenoceptors } \\
\text { in the vasculature, } \\
\text { causing a clinically } \\
\text { significant reduction } \\
\text { in blood pressure as } \\
\text { a result of a reduction } \\
\text { in systemic vascular } \\
\text { resistance }\end{array}$ & $\begin{array}{l}\text { Benign prostatic } \\
\text { hypertrophy }\end{array}$ & - & Orthostatic hypotension \\
\hline $\begin{array}{l}\text { Angiotensin II } \\
\text { antagonists }\end{array}$ & $\begin{array}{l}\text { Azilsartan } \\
\text { Candesartan } \\
\text { Eprosartan } \\
\text { Irbesartan } \\
\text { Losartan } \\
\text { Olmesartan } \\
\text { Telmisartan } \\
\text { Valsartan }\end{array}$ & $\begin{array}{l}\text { Blocks angiotensin II } \\
\text { type I receptors }\end{array}$ & $\begin{array}{l}\text { Diabetes mellitus, post- } \\
\text { Ml patients, patients } \\
\text { with contra-indications } \\
\text { to ACE inhibitors, renal } \\
\text { dysfunction }\end{array}$ & $\begin{array}{l}\text { Pregnancy, } \\
\text { hyperkalaemia, } \\
\text { bilateral renal artery } \\
\text { stenosis }\end{array}$ & - \\
\hline Beta-blockers & $\begin{array}{l}\text { Atenolol } \\
\text { Bisoprolol } \\
\text { Carvedilol } \\
\text { Esmolol } \\
\text { Metoprolol } \\
\text { Nebivolol } \\
\text { Pindolol } \\
\text { Propranolol }\end{array}$ & $\begin{array}{l}\text { Competitively } \\
\text { antagonises effect } \\
\text { of catecholamines at } \\
\beta \text {-adrenergic receptor } \\
\text { sites }\end{array}$ & $\begin{array}{l}\text { Angina, post-MI } \\
\text { patients, congestive } \\
\text { heart failure, glaucoma }\end{array}$ & Asthma, AV block & $\begin{array}{l}\text { Peripheral artery } \\
\text { disease, metabolic } \\
\text { syndrome, glucose } \\
\text { intolerance, physically } \\
\text { active patients, COPD }\end{array}$ \\
\hline $\begin{array}{l}\text { Calcium antagonists } \\
\text { (dihydropyridines) }\end{array}$ & $\begin{array}{l}\text { Amlodipine } \\
\text { Felodipine } \\
\text { Lercanidipine } \\
\text { Nifedipine }\end{array}$ & $\begin{array}{l}\text { Dilates coronary and } \\
\text { peripheral arteries as } \\
\text { a result of inhibition of } \\
\text { cellular } \mathrm{Ca}^{2+} \text { influx }\end{array}$ & Angina, LV hypertrophy & - & $\begin{array}{l}\text { Tachyarrhythmias, heart } \\
\text { failure }\end{array}$ \\
\hline Calcium antagonists & $\begin{array}{l}\text { Diltiazem } \\
\text { Verapamil }\end{array}$ & (ditto) & (ditto) & $\begin{array}{l}\text { AV block, heart } \\
\text { failure }\end{array}$ & - \\
\hline Thiazide diuretics & $\begin{array}{l}\text { Hydrochlorothiazide } \\
\text { Indapamide } \\
\text { Metolazone }\end{array}$ & $\begin{array}{l}\text { Inhibits the } \mathrm{Na}^{+} / \mathrm{Cl}^{-} \\
\text {cotransporter in the distal } \\
\text { tubule }\end{array}$ & $\begin{array}{l}\text { Elderly patients with no } \\
\text { co-morbid conditions }\end{array}$ & Gout & $\begin{array}{l}\text { Metabolic syndrome, } \\
\text { glucose intolerance, } \\
\text { pregnancy }\end{array}$ \\
\hline
\end{tabular}

Abbreviations: $\mathrm{ACE}=$ angiotensin-converting enzyme; $\mathrm{AV}=$ atrioventricular; $\mathrm{COPD}=$ chronic obstructive pulmonary disease; $\mathrm{LV}=$ left ventricular; $\mathrm{MI}=$ myocardial infarction

in the United Kingdom and the United States as an adjunct to diet and maximally tolerated statin therapy for the treatment of individuals with primary hypercholesterolaemia or mixed dyslipidaemia, or those with clinical ASCVD who require additional lowering of LDL-C..$^{38,39}$ Clinical trials have demonstrated decreases in LDL-C by up to $60 \%$ in subjects receiving these agents ${ }^{38}$; definitive evidence of reduced cardiovascular event rates associated with their use may be provided by ongoing trials.

\section{Diabetes}

Risk factor treatment goal: glycated haemoglobin $<7 \%{ }^{20-22}$

- All diabetic patients are considered high risk for the development of ASCVD ${ }^{9,21}$ and should receive appropriate management upon diagnosis. This includes guidance on diet modification and increased physical activity in conjunction with pharmacotherapy. ${ }^{20-22}$ Early initiation of medication is recommended to avoid any delay in treatment. Insulin is administered if treatment goals are not achieved with oral therapy. ${ }^{20-22}$ Treatment goal for glycaemia should be tailored according to the patient profile in order to avoid hypoglycaemia in those with co-morbidities or in elderly patients. ${ }^{22,40}$

- In diabetic patients, treat other ASCVD risk factors more aggressively, ${ }^{22}$ including hypertension. Nevertheless, present evidence suggests that a BP target of $<140 / 85 \mathrm{~mm} \mathrm{Hg}$ is appropriate in patients with diabetes, with a lower 
TABLE 3. Summary of lipid management goals $\mathrm{s}^{6,9,34}$

\begin{tabular}{|c|c|c|c|c|}
\hline & \multicolumn{4}{|c|}{ Recommended lipid levels (mmol/L or \% reduction) } \\
\hline & $\begin{array}{l}2016 \text { European Guidelines on } \\
\text { Cardiovascular Disease Prevention } \\
\text { in Clinical Practice }\end{array}$ & $\begin{array}{l}2013 \text { ACC/AHA Guideline on the } \\
\text { Treatment of Blood Cholesterol } \\
\text { to Reduce Atherosclerotic } \\
\text { Cardiovascular Risk in Adults }\end{array}$ & $\begin{array}{l}2014 \text { NICE Clinical } \\
\text { Guideline on Lipid } \\
\text { Modification }\end{array}$ & $\begin{array}{l}2016 \text { Hong Kong } \\
\text { Cardiovascular } \\
\text { Task Force } \\
\text { Recommendations }\end{array}$ \\
\hline Primary prevention & $\begin{array}{l}\mathrm{LDL}-\mathrm{C}<1.8 \text { to }<3.0 \mathrm{mmol} / \mathrm{L} \text { according } \\
\text { to } \mathrm{SCORE} \text { risk }\end{array}$ & $\begin{array}{l}\downarrow \text { LDL-C } \geq 30 \% \text { if } 10 \text {-year risk } \\
\geq 7.50 \%\end{array}$ & $\downarrow$ Non HDL-C $\geq 40 \%$ & $\begin{array}{l}\mathrm{LDL}-\mathrm{C}<1.8 \mathrm{mmol} / \mathrm{L} \text { to } \\
<3.0 \mathrm{mmol} / \mathrm{L} \text { according } \\
\text { to individual CV risk } \\
\text { level }\end{array}$ \\
\hline Secondary prevention & LDL-C $<1.8 \mathrm{mmol} / \mathrm{L}$ or $\downarrow$ LDL-C $\geq 50 \%$ & $\downarrow L D L-C \geq 50 \%$ & $\downarrow$ Non HDL-C $\geq 40 \%$ & LDL-C $<1.8 \mathrm{mmol} / \mathrm{L}$ \\
\hline $\begin{array}{l}\text { Familial hyper- } \\
\text { cholesterolaemia }\end{array}$ & No recommendation & $\downarrow L D L-C \geq 50 \%$ & $\downarrow$ Non HDL-C $\geq 40 \%$ & LDL-C $<2.5 \mathrm{mmol} / \mathrm{L}$ \\
\hline Diabetes & LDL-C $<1.8 \mathrm{mmol} / \mathrm{L}$ or $\downarrow$ LDL-C $\geq 50 \%$ & $\begin{array}{l}\downarrow L D L-C \geq 50 \% \text { if } 10 \text {-year ASCVD } \\
\text { risk } \geq 7.50 \% \\
\text { or } \\
\downarrow L D L-C \geq 30-50 \%\end{array}$ & $\downarrow$ Non HDL-C $\geq 40 \%$ & $\begin{array}{l}\mathrm{LDL}-\mathrm{C}<1.8 \mathrm{mmol} / \mathrm{L} \text { to } \\
2.5 \mathrm{mmol} / \mathrm{L} \\
\text { According to individual } \\
\mathrm{CV} \text { risk level }\end{array}$ \\
\hline CKD & $\begin{array}{l}\text { Severe: } \\
\text { LDL-C }<1.8 \mathrm{mmol} / \mathrm{L} \text { or } \downarrow \text { LDL-C } \geq 50 \% \\
\text { Moderate: } \\
\text { LDL-C }<2.6 \mathrm{mmol} / \mathrm{L} \text { or } \downarrow \text { LDL-C } \geq 50 \%\end{array}$ & No target specified & $\downarrow$ Non HDL-C $\geq 40 \%$ & $\begin{array}{l}\mathrm{LDL}-\mathrm{C}<1.8 \mathrm{mmol} / \mathrm{L} \text { to } \\
2.5 \mathrm{mmol} / \mathrm{L} \\
\text { According to individual } \\
\text { CV risk level }\end{array}$ \\
\hline
\end{tabular}

Abbreviations: $\mathrm{ACC} / \mathrm{AHA}=$ American College of Cardiology/American Heart Association; $\mathrm{ASCVD}=$ atherosclerotic cardiovascular disease; $\mathrm{CKD}=\mathrm{chronic}$ kidney disease; CV = cardiovascular; HDL-C = high-density lipoprotein cholesterol; LDL-C = low-density lipoprotein cholesterol; NICE = National Institute for Health and Care Excellence; SCORE = Systematic Coronary Risk Evaluation

TABLE 4. Agents recommended for achieving lipid-lowering goals $\mathbf{s}^{6,9,34}$

\begin{tabular}{|c|c|c|c|}
\hline & \multicolumn{3}{|c|}{ Recommended lipid-lowering agents } \\
\hline & $\begin{array}{l}2016 \text { European Guidelines } \\
\text { on Cardiovascular Disease } \\
\text { Prevention in Clinical Practice }^{9}\end{array}$ & $\begin{array}{l}2013 \text { ACC/AHA Guideline on the } \\
\text { Treatment of Blood Cholesterol } \\
\text { to Reduce Atherosclerotic } \\
\text { Cardiovascular Risk in Adults }\end{array}$ & $\begin{array}{l}2014 \text { NICE Clinical Guideline on Lipid } \\
\text { Modification }\end{array}$ \\
\hline Primary prevention & Statin & $\begin{array}{l}10 \text {-Year risk } \geq 7.5 \% \text { : moderate } \\
\text { hight-intensity statin }\end{array}$ & $\begin{array}{l}10-\text { Year risk } \geq 10 \% \text { : atorvastatin } 20 \mathrm{mg} \\
\text { Age } \geq 85 \text { years: consider atorvastatin } 20 \mathrm{mg}\end{array}$ \\
\hline Secondary prevention & Statin & $\begin{array}{l}\leq 75 \text { Years: high-intensity statin } \dagger \\
>75 \text { Years: moderate statin* }\end{array}$ & Atorvastatin $80 \mathrm{mg}$ \\
\hline $\begin{array}{l}\text { Familial hyper- } \\
\text { cholesterolaemia }\end{array}$ & Statin & High-intensity statin $†$ & $\begin{array}{l}\text { Atorvastatin } 20 \mathrm{mg} \\
\text { Consider ezetimibe for } \mathrm{FH}\end{array}$ \\
\hline Diabetes & Statin & $\begin{array}{l}10 \text {-Year risk } \geq 7.5 \% \text { : high-intensity statin } \dagger \\
10 \text {-Year risk }<7.5 \% \text { : moderate-intensity } \\
\text { statin }^{\star}\end{array}$ & $\begin{array}{l}\text { Type 1: consider atorvastatin } 20 \mathrm{mg} \\
\text { Type } 2 \text { and } 10 \text {-year risk } \geq 10 \% \text { : atorvastatin } \\
20 \mathrm{mg}\end{array}$ \\
\hline CKD & Statin & No specific recommendation & $\begin{array}{l}\text { Atorvastatin } 20 \mathrm{mg} \\
\text { If non-HDL-C } \downarrow<40 \% \text { : consider higher } \\
\text { dose }\end{array}$ \\
\hline $\begin{array}{l}\text { Combination with } \\
\text { non-statin }\end{array}$ & $\begin{array}{l}\text { If target not reached with highest } \\
\text { tolerated statin }\end{array}$ & $\begin{array}{l}\text { If target not reached with highest } \\
\text { tolerated statin }\end{array}$ & $\begin{array}{l}\text { Do not offer fibrate, niacin, BAS, or } \\
\text { omega- } 3 \text { fatty acid }\end{array}$ \\
\hline
\end{tabular}

Abbreviations: ACC/AHA = American College of Cardiology/American Heart Association; BAS = bile acid sequestrant; $\mathrm{CKD}=$ chronic kidney disease; $\mathrm{FH}=$ familial hypercholesterolaemia; HDL-C = high-density lipoprotein cholesterol; LDL-C = low-density lipoprotein cholesterol; NICE = National Institute for Health and Care Excellence

* Moderate-to-high-intensity statin: Expected to reduce LDL-C by 30\%-50\%, eg atorvastatin 10-20 mg, rosuvastatin 5- 10 mg, simvastatin $20-40$ mg, pravastatin $40-80 \mathrm{mg}$, lovastatin $40 \mathrm{mg}$

† High-intensity statin: Expected to reduce LDL-C by $\geq 50 \%$, eg atorvastatin $40-80$ mg, rosuvastatin $20-40 \mathrm{mg}$

BP (systolic BP of $<130 \mathrm{~mm} \mathrm{Hg}$ ) as an option in patients with hypertension and nephropathy. It should be noted that lower BP may be associated with increased risk of adverse events, especially in older patients or those with a long duration of diabetes, and the risk and benefit of intensive BP lowering needs to be considered individually according to the patient profile. ${ }^{22}$ 
TABLE 5. Classes of lipid-lowering agents

\begin{tabular}{|c|c|c|c|c|c|}
\hline Drug class & $\begin{array}{l}\text { Agents available } \\
\text { in Hong Kong }\end{array}$ & Mechanism of action & $\begin{array}{l}\text { Conditions favouring } \\
\text { use of drug class }\end{array}$ & $\begin{array}{l}\text { Compelling contra- } \\
\text { indications }\end{array}$ & $\begin{array}{l}\text { Possible contra- } \\
\text { indications }\end{array}$ \\
\hline Statins & $\begin{array}{l}\text { Atorvastatin } \\
\text { Fluvastatin } \\
\text { Lovastatin } \\
\text { Pravastatin } \\
\text { Rosuvastatin } \\
\text { Simvastatin }\end{array}$ & $\begin{array}{l}\text { Inhibit the enzyme HMG- } \\
\text { CoA reductase, which } \\
\text { plays a central role in } \\
\text { cholesterol synthesis }\end{array}$ & $\begin{array}{l}\text { Existing } \\
\text { cardiovascular } \\
\text { disease, very high } \\
\text { LDL-C levels, } \\
\text { diabetes, patients with } \\
\text { a high 10-year risk } \\
\text { of Ml }\end{array}$ & $\begin{array}{l}\text { Should not be used in } \\
\text { patients with active or } \\
\text { chronic liver disease, or } \\
\text { by pregnant or lactating } \\
\text { women }\end{array}$ & $\begin{array}{l}\text { Liver function should } \\
\text { be tested before, } \\
\text { after } 6-12 \text { weeks of } \\
\text { therapy, and with } \\
\text { any dose increase. } \\
\text { Advise patient to } \\
\text { consult immediately if } \\
\text { signs of muscle pain } \\
\text { suggesting myopathy } \\
\text { occur }\end{array}$ \\
\hline Fibrates & $\begin{array}{l}\text { Bezafibrate } \\
\text { Fenofibrate } \\
\text { Gemfibrozil }\end{array}$ & $\begin{array}{l}\text { Varies depending on } \\
\text { the agent. Bezafibrate, } \\
\text { fenofibrate reduce the } \\
\text { concentration of VLDL } \\
\text { by increasing the activity } \\
\text { of lipoprotein lipase. } \\
\text { Gemfibrozil inhibits } \\
\text { lipolysis, reduces hepatic } \\
\text { fatty acid uptake, and } \\
\text { reduces secretion of VLDL } \\
\text { from the liver }\end{array}$ & $\begin{array}{l}\text { Very high levels of } \\
\text { plasma triglycerides }\end{array}$ & $\begin{array}{l}\text { Not recommended in } \\
\text { patients with severe renal } \\
\text { or hepatic impairment, } \\
\text { hypoalbuminaemia, } \\
\text { primary biliary cirrhosis, } \\
\text { gallbladder disease, or } \\
\text { nephrotic syndrome. } \\
\text { Should not be used by } \\
\text { pregnant or lactating } \\
\text { women }\end{array}$ & $\begin{array}{l}\text { Use with caution in } \\
\text { mild-to-moderate renal } \\
\text { impairment; monitor } \\
\text { serum transaminase } \\
\text { levels regularly. } \\
\text { May cause myalgia, } \\
\text { myopathy. } \\
\text { Rarely used with } \\
\text { statins, may interact } \\
\text { with anticoagulant } \\
\text { therapy }\end{array}$ \\
\hline $\begin{array}{l}\text { Bile-acid sequestrants } \\
\text { (resins) }\end{array}$ & Cholestyramine & $\begin{array}{l}\text { Binds with bile acids } \\
\text { to form an insoluble } \\
\text { complex that is excreted } \\
\text { in the faeces. The } \\
\text { increasing loss of bile acid } \\
\text { increases the oxidation of } \\
\text { cholesterol to bile acids }\end{array}$ & $\begin{array}{l}\text { Patients for whom } \\
\text { statin therapy is } \\
\text { contra-indicated or } \\
\text { those who cannot } \\
\text { tolerate statin therapy }\end{array}$ & $\begin{array}{l}\text { Contra-indicated } \\
\text { in complete biliary } \\
\text { obstruction, high fasting } \\
\text { triglycerides }\end{array}$ & $\begin{array}{l}\text { May decrease folate } \\
\text { levels }\end{array}$ \\
\hline $\begin{array}{l}\text { Selective cholesterol } \\
\text { absorption inhibitor }\end{array}$ & Ezetimibe & $\begin{array}{l}\text { Localises in the brush } \\
\text { border of the small } \\
\text { intestine where it inhibits } \\
\text { absorption of cholesterol, } \\
\text { decreasing its delivery to } \\
\text { the liver. This decrease in } \\
\text { cholesterol stored in the } \\
\text { liver increases cholesterol } \\
\text { clearance from the blood }\end{array}$ & $\begin{array}{l}\text { Patients for whom } \\
\text { statin therapy is } \\
\text { contra-indicated or } \\
\text { those who cannot } \\
\text { tolerate statin therapy }\end{array}$ & $\begin{array}{l}\text { Active liver disease. } \\
\text { Should not be used by } \\
\text { pregnant or lactating } \\
\text { women }\end{array}$ & $\begin{array}{l}\text { Use with caution in } \\
\text { patients with renal or } \\
\text { hepatic impairment }\end{array}$ \\
\hline $\begin{array}{l}\text { Combination } \\
\text { cholesterol absorption } \\
\text { inhibitor and statin }\end{array}$ & $\begin{array}{l}\text { Ezetimibe- } \\
\text { simvastatin }\end{array}$ & $\begin{array}{l}\text { Simvastatin inhibits the } \\
\text { HMG-CoA reductase } \\
\text { enzyme and thus } \\
\text { cholesterol synthesis, } \\
\text { while ezetimibe inhibits } \\
\text { cholesterol absorption } \\
\text { in the small intestine, } \\
\text { reducing liver cholesterol } \\
\text { stores and increasing } \\
\text { cholesterol clearance from } \\
\text { the blood }\end{array}$ & $\begin{array}{l}\text { Patients who } \\
\text { cannot achieve } \\
\text { target LDL-C levels } \\
\text { despite receiving the } \\
\text { maximum tolerated } \\
\text { statin dose }\end{array}$ & $\begin{array}{l}\text { Contra-indications } \\
\text { include hypersensitivity, } \\
\text { active liver disease or } \\
\text { unexplained persistent } \\
\text { elevations of serum } \\
\text { transaminases, } \\
\text { pregnancy and lactation, } \\
\text { and concomitant } \\
\text { administration of potent } \\
\text { CYP3A4 inhibitors, } \\
\text { gemfibrozil, cyclosporine } \\
\text { or danazol }\end{array}$ & - \\
\hline Nicotinic acid & Nicotinic acid & $\begin{array}{l}\text { The exact mechanisms } \\
\text { are currently unknown, but } \\
\text { are not related to its role } \\
\text { as a vitamin }\end{array}$ & $\begin{array}{l}\text { Patients who cannot } \\
\text { tolerate statin therapy, } \\
\text { and for those with } \\
\text { very low levels of } \\
\text { HDL-C and/or high } \\
\text { triglyceride levels }\end{array}$ & $\begin{array}{l}\text { Contra-indications include } \\
\text { hypersensitivity reactions } \\
\text { to nicotinic acid, liver } \\
\text { disease, impaired glucose } \\
\text { tolerance, diabetes } \\
\text { mellitus, and gouty } \\
\text { arthritis }\end{array}$ & - \\
\hline
\end{tabular}

Abbreviations: HDL-C = high-density lipoprotein cholesterol; HMG-CoA = 3-hydroxy-3-methyl coenzyme A; LDL-C = low-density lipoprotein cholesterol; $\mathrm{MI}=$ myocardial infarction; VLDL = very-low-density lipoproteins

\section{Discussion}

The gap between evidence and practice

Although clear, evidence-based guidelines and For example, Yusuf et $\mathrm{al}^{43}$ reported worldwide poor recommendations for ASCVD prevention have use of medications for the secondary prevention of 
ASCVD. Their study included 153996 adults aged 35 to 70 years from rural and urban communities in high-, upper-middle-, lower-middle-, and low-income countries, 5650 of whom had had a self-reported CHD event and 2292 a stroke. Few individuals with ASCVD took antiplatelet drugs (25.3\%), $\beta$-blockers (17.4\%), ACE inhibitors or ARBs (19.5\%), or statins (14.6\%). As expected, drug use was higher in high-income countries, with $11.2 \%$ of patients in these countries not receiving any drugs compared with $45.1 \%$ of patients in upper-middleincome countries, $69.3 \%$ in lower-middle-income countries, and $80.2 \%$ in low-income countries. Notably, despite the relative accessibility of drugs for secondary prevention of ASCVD in high- and upper-middle-income countries, many patients remained untreated.

Of the patients who do receive treatment for ASCVD risk factors, only a few attain their treatment goals. Findings from the Hong Kong Cardiovascular Task Force Risk Management Programme indicate that $84 \%$ of enrolled hypertensive patients were treated with one or two antihypertensive drugs, most commonly ARBs (63.5\%) and calcium channel blockers (47.2\%; BMY Cheung, unpublished data). Similarly $64 \%$ of the diabetic patients were treated with metformin $(68.8 \%)$ and/or gliptins (36\%), while $78.1 \%$ of patients with dyslipidaemia were treated with a statin. Notably, however, treatment goals for hypertension $(<130 / 80 \mathrm{~mm} \mathrm{Hg}$ for diabetic patients, $<140 / 90 \mathrm{~mm} \mathrm{Hg}$ for non-diabetics) and diabetes (glycated haemoglobin $<7 \%$ ) were met by just over $50 \%$ of hypertensive patients and approximately $60 \%$ of diabetics.

Ensuring physician compliance with evidence-based guidelines and improving clinician understanding of factors affecting patient compliance with treatment may be the key to decreasing ASCVD risk in the Hong Kong population.

\section{Differences between the 2008 and 2016 consensus statements}

The present update of the 2008 Consensus Statement introduces the use of the new American Pooled Cohort Risk Assessment Equations that have superseded the Framingham Risk Evaluation; ASCVD risk can be assessed using either these equations or the European SCORE system to stratify patients into low-, moderate-, or high-risk categories to aid targeting of therapies as well as the establishment of suitable treatment goals. The risk factor reduction goals for hypertension and dyslipidaemia have been updated to reflect the most current recommendations from the Eighth Joint National Committee, the European guidelines on the management of arterial hypertension, and the European guidelines on cardiovascular disease prevention in clinical practice. The HDL-C target included in the 2008 Consensus Statement has been omitted from the current update as increased $\mathrm{HDL}-\mathrm{C}$ has not been proven to reduce ASCVD risk.

\section{Conclusions}

The development of ASCVD in at-risk patients may be slowed and/or prevented by lifestyle modification, reduction of metabolic risk factors, and pharmacological treatment. The clinician plays a central role in ASCVD prevention-identifying at-risk patients, calculating the total ASVCD risk score, encouraging lifestyle changes, and providing targeted interventions to achieve specific treatment goals. Nonetheless, it is vital that the clinician is not overly focused on the treatment of isolated ASCVD risk factors but should instead adopt a 'wholeperson' approach to diagnosis and therapy. Many patients present with multiple risk factors and, therefore, individualised, nuanced patient evaluation and management is essential to achieve optimum outcomes. Finally, none of these interventions will result in ASVCD prevention without the cooperation of the patient. Clinicians are encouraged to build strong partnerships with their patients, with the aim of establishing individual ownership of their treatment plans and, thus, improved treatment compliance.

\section{Acknowledgements}

The authors would like to acknowledge Ms Lianne Cowie and Dr Jose Miguel (Awi) Curameng of MIMS (Hong Kong) Limited for providing editorial and writing support, which was funded by Pfizer Corporation Hong Kong Limited. The meetings during which these consensus points were formulated and discussed were supported by an unrestricted educational grant from Pfizer Corporation Hong Kong Limited.

\section{Declaration}

Source of support: Editorial and writing services, and the meetings during which these consensus points were formulated and discussed, were supported by an unrestricted educational grant from Pfizer Corporation Hong Kong Limited.

\section{References}

1. Goff DC Jr, Lloyd-Jones DM, Bennett G, et al. 2013 ACC/ AHA guideline on the assessment of cardiovascular risk: a report of the American College of Cardiology/American Heart Association Task Force on Practice Guidelines. Circulation 2014;129(25 Suppl 2):S49-73.

2. Yusuf S, Reddy S, Ounpuu S, Anand S. Global burden of cardiovascular diseases. Part I: general considerations, the epidemiologic transition, risk factors, and impact of urbanization. Circulation 2001;104:2746-53.

3. Cheung MY, Ong KL, Tso WK, Lam TH, Lam SL. Increasing 
prevalence of hypertension in Hong Kong Cardiovascular Risk Factor Prevalence Study: role of general and central obesity. Proceedings of the 16th Medical Research Conference; 2011 Jan 22; Department of Medicine, The University of Hong Kong, Hong Kong. Hong Kong Med J 2011;17(Suppl 1):16S.

4. Roth GA, Huffman MD, Moran AE, et al. Global and regional patterns in cardiovascular mortality from 1990 to 2013. Circulation 2015;132:1667-78.

5. Cheung MY, Chow CC, Chu DW, et al. The Hong Kong Cardiovascular Task Force. Towards cardiovascular disease protection. Consensus statement on preventing cardiovascular disease in the Hong Kong population. Med Prog 2008;35:473-9.

6. Stone NJ, Robinson JG, Lichtenstein AH, et al. 2013 ACC/ AHA guideline on the treatment of blood cholesterol to reduce atherosclerotic cardiovascular risk in adults: a report of the American College of Cardiology/American Heart Association Task Force on Practice Guidelines. J Am Coll Cardiol 2014;63(25 Pt B):2889-934.

7. Mancia G, Fagard R, Narkiewicz K, et al. 2013 ESH/ESC guidelines for the management of arterial hypertension: the Task Force for the Management of Arterial Hypertension of the European Society of Hypertension (ESH) and of the European Society of Cardiology (ESC). Eur Heart J 2013;34:2159-219.

8. James PA, Oparil S, Carter BL, et al. 2014 Evidence-based guideline for the management of high blood pressure in adults: report from the panel members appointed to the Eighth Joint National Committee (JNC 8). JAMA 2014;311:507-20.

9. Piepoli MF, Hoes AW, Agewall S, et al. 2016 European guidelines on cardiovascular disease prevention in clinical practice: The Sixth Joint Task Force of the European Society of Cardiology and Other Societies on Cardiovascular Disease Prevention in Clinical Practice (constituted by representatives of 10 societies and by invited experts) developed with the special contribution of the European Association for Cardiovascular Prevention \& Rehabilitation (EACPR). Eur Heart J 2016;37:2315-81.

10. Collins GS, Altman DG. Predicting the 10 year risk of cardiovascular disease in the United Kingdom: independent and external validation of an updated version of QRISK2. BMJ 2012;344:e4181.

11. QRISK2. Available from: https://www.qrisk.org/2016/. Accessed 9 Jan 2017.

12. Scandinavian Simvastatin Survival Group. Randomized trial of cholesterol lowering in 4,444 patients with coronary heart disease: the Scandinavian Simvastatin Survival Study (4S). Lancet 1994;344:1383-9.

13. Shepherd J, Cobbe SM, Ford I, et al. Prevention of coronary heart disease with pravastatin in men with hypercholesterolemia. West of Scotland Coronary Prevention Study Group. N Engl J Med 1995;333:1301-7.

14. Intensive blood-glucose control with sulphonylureas or insulin compared with conventional treatment and risk of complications in patients with type 2 diabetes (UKPDS 33). UK Prospective Diabetes Study (UKPDS) Group. Lancet 1998;352:837-53.

15. Retinopathy and nephropathy in patients with type 1 diabetes four years after a trial of intensive therapy. The Diabetes Control and Complications Trial/Epidemiology of Diabetes Interventions and Complications Research
Group. N Engl J Med 2000;342:381-9.

16. Antithrombotic Trialists' Collaboration. Collaborative meta-analysis of randomised trials of antiplatelet therapy for prevention of death, myocardial infarction, and stroke in high risk patients. BMJ 2002;324:71-86.

17. US Department of Health and Human Services. The health benefits of smoking cessation. Washington DC: US Department of Health and Human Services; 1990.

18. Taylor R, Brown A, Ebrahim S, et al. Exercise-based rehabilitation for patients with coronary heart disease: systematic review and meta-analysis of randomized controlled trials. Am J Med 2004;116:682-92.

19. Prevention of cardiovascular disease. Guidelines for assessment and management of cardiovascular risk. Geneva, World Health Organization; 2007.

20. American Diabetes Association. Standards of medical care in diabetes-2014. Diabetes Care 2014;37(Suppl 1):S1480.

21. The Hong Kong reference framework for diabetes care for adults in primary care settings. Available from: http:// www.pco.gov.hk/english/resource/files/RF_DM_full.pdf. Accessed 1 Dec 2014.

22. Rydén L, Grant PJ, Anker SD, et al. ESC guidelines on diabetes, pre-diabetes, and cardiovascular diseases developed in collaboration with the EASD: the Task Force on diabetes, pre-diabetes, and cardiovascular diseases of the European Society of Cardiology (ESC) and developed in collaboration with the European Association for the Study of Diabetes (EASD). Eur Heart J 2013;34:3035-87.

23. Wang C, Collet JP, Lau J. The effect of Tai Chi on health outcomes in patients with chronic conditions: a systematic review. Arch Intern Med 2004;164:493-501.

24. WHO Expert Consultation. Appropriate body-mass index for Asian populations and its implications for policy and intervention strategies. Lancet 2004;363:157-63.

25. International Diabetes Federation. The IDF consensus worldwide definition of the metabolic syndrome. Available from: http://www.idf.org/webdata/docs/MetSyndrome_ FINAL.pdf. Accessed 10 Jul 2015.

26. West R, McNeill A, Raw M. Smoking cessation guidelines for health professionals: an update. Health Education Authority. Thorax 2000;55:987-99.

27. National Institute for Health and Clinical Excellence. Hypertension in adults: diagnosis and management. NICE guideline (CG127). August 2011. Available from: https:// www.nice.org.uk/guidance/cg127. Accessed 10 Jul 2015.

28. National Institute for Health and Clinical Excellence. Type 2 diabetes: The management of type 2 diabetes. NICE guideline (CG87). May 2009. Accessed 10 Jul 2015.

29. National Institute for Health and Care Excellence. Chronic kidney disease in adults: assessment and management. NICE guideline (CG182). July 2014. Accessed 10 Jul 2015.

30. Shen L, Shah BR, Reyes EM, et al. Role of diuretics, $\beta$ blockers, and statins in increasing the risk of diabetes in patients with impaired glucose tolerance: reanalysis of data from the NAVIGATOR study. BMJ 2013;347:f6745.

31. Bangalore S, Parkar S, Grossman E, Messerli FH. A metaanalysis of 94,492 patients with hypertension treated with beta blockers to determine the risk of new-onset diabetes mellitus. Am J Cardiol 2007;100:1254-62.

32. Messerli FH, Bangalore S. Half a century of hydrochlorothiazide: facts, fads, fiction, and follies. Am J Med 2011;124:896-9. 
33. Williams B, MacDonald TM, Morant S, et al. Spironolactone versus placebo, bisoprolol, and doxazosin to determine the optimal treatment for drug-resistant hypertension (PATHWAY-2): a randomised, double-blind, crossover trial. Lancet 2015;386:2059-68.

34. National Institute for Health and Care Excellence. Cardiovascular disease: risk assessment and reduction, including lipid modification. NICE guideline (CG181). July 2014. Accessed 10 Jul 2015.

35. Baigent C, Keech A, Kearney PM, et al. Efficacy and safety of cholesterol-lowering treatment: prospective metaanalysis of data from 90056 participants in 14 randomised trials of statins. Lancet 2005;366:1267-78.

36. Tomlinson B, Hu M, Zhang Y, Liu ZM, Chan P. Response to the letter "No evidence to support high-intensity statin in Chinese patients with coronary heart disease". Int J Cardiol 2016;209:192-3

37. Liao JK. Safety and efficacy of statins in Asians. Am J Cardiol 2007;99:410-4.

38. Everett BM, Smith RJ, Hiatt WR. Reducing LDL with
PCSK9 inhibitors-the clinical benefit of lipid drugs. N Engl J Med 2015;373:1588-91.

39. Mayor S. NICE recommends PCSK9 inhibitors for patients not responding to statins. BMJ 2016;353:i2609.

40. Inzucchi SE, Bergenstal RM, Buse JB, et al. Management of hyperglycemia in type 2 diabetes, 2015: a patient-centered approach: update to a position statement of the American Diabetes Association and the European Association for the Study of Diabetes. Diabetes Care 2015;38:140-9.

41. Ma J, Sehgal NL, Ayanian JZ, Stafford RS. National trends in statin use by coronary heart disease risk category. PLoS Med 2005;2:e123.

42. Pearson TA. The prevention of cardiovascular disease: have we really made progress? Health Aff (Millwood) 2007;26:49-60.

43. YusufS, Islam S, Chow CK, et al. Use of secondary prevention drugs for cardiovascular disease in the community in highincome, middle-income, and low-income countries (the PURE Study): a prospective epidemiological survey. Lancet 2011;378:1231-43. 